\title{
Glimpses of 3D Vector based Membrane Photolithography
}

\author{
Sheetal S. Patil \\ Department of Computer Engineering, Bharati Vidyapeeth University College of Engineering, Pune \\ sspatil@bvucoep.edu.in
}

\begin{tabular}{|l|l|}
\hline Article History & \multicolumn{1}{|c|}{ Abstract } \\
$\begin{array}{l}\text { Article Submission } \\
\text { 24 October 2021 } \\
\text { Revised Submission } \\
11 \text { November 2021 } \\
\text { Article Accepted } \\
10 \text { December 2021 } \\
\text { Article Published } \\
20 \text { January 2022 }\end{array} \quad \begin{array}{l}\text { In optical lithography, decline in the profundity of centre has become an intense } \\
\text { issue, alongside the trouble in improving goal. In memory chip creation, the } \\
\text { issues. Notwithstanding PSM innovation, some super-goal lithographic applications } \\
\text { have likewise been proposed. In the later methodology, no PSMs are required, in this } \\
\text { manner a portion of the challenges concerning cover innovation can be maintained a } \\
\text { strategic distance from. Be that as it may, it isn't in every case simple to apply PSM } \\
\text { or super-goal advancements to muddled cover designs, for example, for ASIC, chip } \\
\text { rationale IC's. For fine occasional examples, a high spatial frequency upgrading } \\
\text { channel is utilized with annular enlightenment. With sideways occurrence } \\
\text { enlightenments, light shafts which are significantly diffracted by veil examples can go } \\
\text { through the viewpoint student, in this way, the band-width of the spatial frequency } \\
\text { transmission qualities of the focal point framework is expanded. The student channel } \\
\text { smothers the transmission of direct bars concerning the diffracted pillars, improving } \\
\text { the picture differentiates in the high frequency locale. Thorough re-enactments run } \\
\text { rapidly on a workstation for complex 20 customary and stage moving covers, } \\
\text { substrate bleaching, and optical metrology and arrangement issues. } \\
\text { Keywords: Optical lithography, ASIC, bleaching, spatial frequency }\end{array}$ \\
\hline
\end{tabular}

\section{Introduction}

The pupil separating focal point framework comprises of two kinds of understudy channels and two sorts of brightening designs. These channels are chosen relying upon the cover example to be utilized. For fine window designs, the super-FLEX channel is utilized with customary enlightenment. We have indicated that the DOF and goal for window examples can be improved by presenting a particular sort of stage adequacy adjustment 161 into the wave front at the focal point student. By superposing the sufficiency of two pictures with various central planes, and controlling the separation and the stage connection between them, a joined impact of the FLEX strategy (different central plane introduction) and edge-improving PSM is acquired. We consider this impact the super-FLEX impact.

Hence, a basic stage channel, whose plentifulness transmission dispersion is appeared in Fig.2, can be utilized. For fine intermittent examples, a high spatial frequency upgrading channel is utilized with annular brightening. It was discovered that this impact is acquired by changing the period of the light going through the focal piece of the understudy. The capacity to configuration designed materials that show a variety of physical, concoction, and organic capacities utilizing standard photolithographic strategies is giving new high through-put approaches in sensor microarrays, genomics, sedate screening, proteomics research, and even materials amalgamation. Stretching out this technique to fluidic layer works that require helpful elements and wet situations is attractive to comprehend, copy, example, and adventure numerous elements of cell films for central biophysical research and numerous biomedical and detecting innovations.

We have as of late built up another optical device (wet photolithography) to draw bio mimetic liquids lowered in watery conditions. In an early showing, they made a variety of stable gaps inside a phospholipid's bilayer - an engineered copy that catches numerous properties of cell layers - just by designed brightening of tests utilizing profound bright light. 


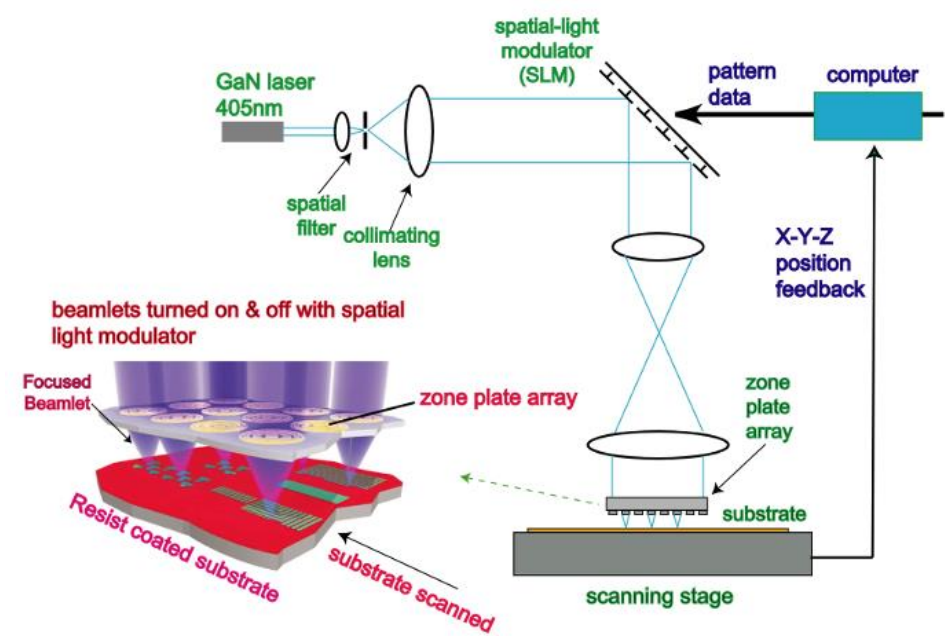

Figure 1: 3D Optical Photolithography

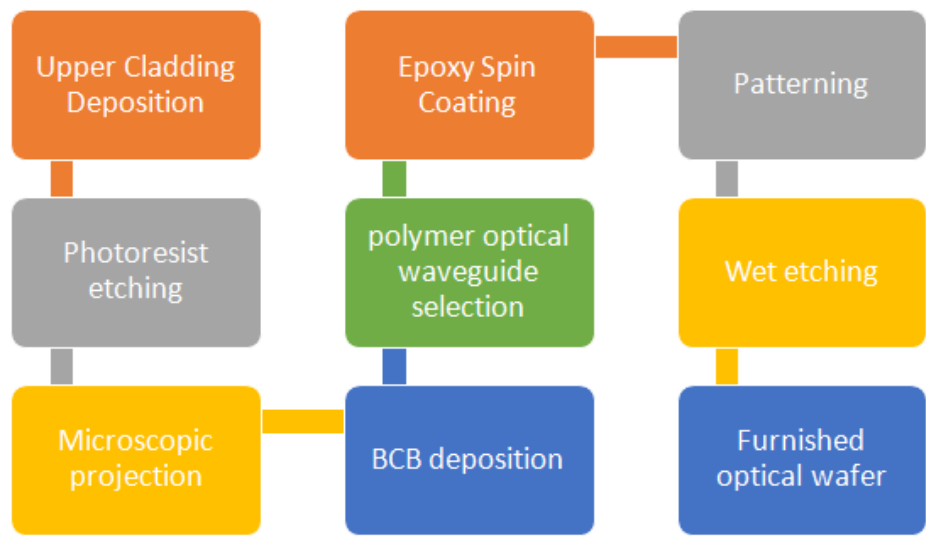

Figure 2: Process flow in 3D Optical Photolithography

The waveguide technique has the benefit of being a lot quicker than limited component or limited contrast strategies at taking care of the light dissipating issue. The waveguide strategy is a spatial frequency area technique which normally permits thorough recreation of non-vertical brightening, defocus, huge numerical gap (NA) focal points, both light polarization modes and broadened source incomplete intelligence. Maxwell's conditions are comprehended for every heading of approaching light. No approximations for registering the dissipating of non-vertically occurrence light is vital.

\section{References}

[1] C. K. Yee, M. L. Amweg, A. R. Sapuri, M. C. Howland and A. N. Parikh, "Membrane photolithography," Digest of the LEOS Summer Topical Meetings Biophotonics/Optical Interconnects and VLSI Photonics/WBM Microcavities, 2004., San Diego, CA, USA, 2004, pp. 2 pp.-, doi: 10.1109/LEOSST.2004.1338659.

[2] K. Prasad, "A generic computer simulation model to characterize photolithography manufacturing area in an IC fab facility," Ninth IEEE/CHMT International Symposium on Electronic Manufacturing Technology, Competitive Manufacturing for the Next Decade, Washington, DC, USA, 1990, pp. 6672, doi: 10.1109/IEMT9.1990.114982.

[3] T. Prevenslik, "EFM and ESD in photolithography by cavity QED," Fourtieth IAS Annual Meeting. Conference Record of the 2005 Industry Applications Conference, 2005., Kowloon, Hong Kong, 2005, pp. 1103-1107 Vol. 2, doi: 10.1109/IAS.2005.1518494. 